\title{
Usabilidad del sistema colaborativo Groove Networks y el sistema de teleenseñanza como soporte para cursos en Internet
}

Recepción: Agosto de 2007 / Aceptación: Noviembre de 2007

${ }^{(1)}$ Gerber Incacari Sancho (2) Rosmeri Mayta Huatuco

\begin{abstract}
RESUMEN
En el presente artículo se ha determinado la usabilidad del sistema colaborativo Groove Networks y el sistema de teleenseñanza como soporte para cursos por Internet. Para este trabajo se utilizó el test de usabilidad que permitió determinar la facilidad de uso del sistema colaborativo, para ello se implementó la plataforma digital del sistema de teleenseñanza (Moodle), para el proceso de enseñanza que se realizó en dos fases: síncrono para Groove Networks y asíncrono para el sistema de teleenseñanza, las cuales se realizaron en un periodo de cuatro semanas, llegando a la conclusión de que la plataforma digital del sistema de teleenseñanza permite llegar a la mayor cantidad de personas y estar presente 24/7(24 horas del día y los siete días de la semana).
\end{abstract}

Palabras clave: Sistema colaborativo, Teleenseñanza, Usabilidad, Herramientas web.

USABILITY SYSTEM COLLABORATIVE GROOVE NETWORKS AND TELEEDUCATION SYSTEM AS A SUPPORT FOR COURSES ON THE INTERNET

\section{ABSTRACT}

In this article has been determined usability of the system collaborative Groove Networks and tele-education system as a support for courses over the Internet. For this study we used the usability test that mapped the ease of use of collaborative system, this was implemented the digital platform system telelearning (Moodle), in the process of teaching that is conducted in two phases: Synchronous for Groove Networks and asynchronous for tele-education system, which were conducted over a period of four weeks, coming to the conclusion that the digital platform for tele-education system leads to the greatest number of people and be present 24 / 7 ( 24 hours the day and seven days a week)

Key words: Collaborative System, Teleenseñanza, Usability, Web Tools.

\section{PLANTEAMIENTO Y DEFINICIONN DEL PROBLEMA}

La tecnología constituye un elemento clave para el desarrollo de la educación y la formación; por el momento es el único medio capaz de satisfacer la demanda de un número de personas que necesita mejorar su capacidad, sobre todo relacionado con el empleo. Aprender es la más importante actividad de información, la enseñanza necesita ser mucho más flexible que faciliten la interacción a distancia entre miembros de un equipo de trabajo, que les permitan coordinar sus actividades de trabajo y de aprendizaje (sistemas de teleenseñanza), programas (software) que les ayuden a distintas personas en la elaboración de un mismo documento electrónico, de hacer más fácil, el proceso de compartir información entre un grupo disperso geográficamente para realizar una tarea en común y que estas sean fáciles de acceder permitiendo la integración dentro de otras actividades, sea en grupo o individualmente (sistemas colaborativo), es decir que los programas deben ser usables, un programa se considera fácil de aprender y de usar en términos del tiempo que toma el usuario para llevar a cabo su objetivo.

Es así que se plantea la siguiente interrogante : ¿cómo es la usabilidad del sistema colaborativo Groove Networks y el sistema de teleenseñanza como soporte para cursos por Internet?

\section{OBJETIVOS DE LAINVESTIGACIÓN}

\section{Objetivo general}

Determinar el nivel de usabilidad del sistema colaborativo Groove Networks y del sistema de teleenseñanza como soporte para cursos por Internet.

\section{Objetivo específicos}

- Implementar la plataforma digital para el sistema de teleenseñanza (Moodle).

- Desarrollar el contenido del curso de Microsoft Word

- Realizar las pruebas de usabilidad del sistema Groove Networks y del sistema de teleenseñanza

\section{JUSTIFICACIÓN}

La mayoría de los métodos de evaluación de sistemas se basan en la funcionalidad de los sistemas y no en la facilidad de uso, se puede presentar programas de gran sofisticación que proporciona solución a problemas complejos, indudablemente si estos no son fáciles de usar

\footnotetext{
1) Ingeniero de Sistemas e Informática, UNMSM

E-mail: isgerber@gmail.com

Ingeniero Industrial. Profesora del Departamento de Sistemas e Informática, UNMSM

E-mail:rmaytah@unmsm.edu.pe
} 
no se podrá lograr los objetivos en los tiempos establecidos, generando gastos innecesarios a la institución, de ello se desprende la necesidad de realizar estudios de usabilidad de sistemas así como de sitios Web.

\section{LATELEENSEÑANZA}

Se designa como teleenseñanza (enseñanza a distancia, enseñanza abierta) a todos los procesos de formación que emplean tecnologías de la comunicación, tiene las siguientes características:

- El alumno y el tutor se encuentran en distintas lugares.

- Es un sistema de aprendizaje flexible, permite al receptor decidir el momento en que se produce, pero es a la vez interactivo porque permite intercambio de información entre profesores y alumnos.

- El medio utilizado son las redes de comunicación.

\section{Modalidades de teleenseñanza}

Desde un punto de vista pedagógico podríamos considerar dos grandes modalidades.

\section{La autoformación tutorizada}

Su metodología es similar a la enseñanza por correspondencia. El alumno recibe una documentación preparada para la autoformación y debe realizar unos ejercicios que debe enviar periódicamente a un tutor al que puede realizar consultas cuando lo desee. Esta modalidad permite que el alumno avance el curso de acuerdo a su capacidad, pero le exige una autodisciplina.

\section{La clase virtual}

Su metodología se basa en la consecución de los objetivos formativos por la interacción profesoralumno y entre alumnos, de modo que se consigan los aspectos motivacionales que se dan en una clase presencial. La ventaja para el alumno que utiliza clase virtual son la adaptación del estudio a sus horarios y la participación más reflexiva, el alumno se sienta en un ambiente tranquilo, para seguir el ritmo de trabajo. Las instituciones que optan por esta metodología de formación eliminan los costos administrativos como mantenimiento de local, equipos etc.

\section{Sistema colaborativo}

Muchas de las características de la sociedad son adquiridas de las formas en que la gente interactúa. Aunque la presencia de computadoras en las casas y oficinas es cada día más común, la interacción entre las personas no dista mucho respecto a como lo hacía una década atrás. Según Jay, A. 1993 señala que los trabajadores de una oficina emplean entre el $30 \%$ y el $70 \%$ de su tiempo en reuniones. Por ejemplo, en una empresa se hacen grupos de personas como planificación, presupuestos, producción, ventas y otros. Sin embargo las aplicaciones de software tienden a ignorar este hecho, creando ambientes de trabajo individuales y que es el único usuario en el computador.

\section{Características}

Dentro de un esquema común de trabajo en grupo, generalmente se establecen roles entre los miembros, ya sean implícitos o explícitos, para hacer más eficiente y coordinado el logro de los objetivos.

\section{Clasificación}

a. Clasificación de tiempo y espacio

Esta clasificación está basada en las nociones de tiempo y espacio. Según, Ellis,C.A2001 define a Groupware como un apoyo a un grupo que actúa cara a cara o a un grupo que trabaja físicamente distribuido. Pero también groupware es considerado como un sistema que ayuda a un grupo que interactúa en tiempo real o en forma asíncrono.

\section{b. Clasificación de nivel de aplicación}

Otra clasificación está basada en el nivel de funcionalidad de la aplicación, también llamada de dominio de aplicación. A esta clasificación pertenecen los sistemas de mensajes, editores multiusuarios, sistemas de apoyo a las decisiones grupales y salas de reuniones electrónicas, conferencias por computador en tiempo real, teleenseñanza, conferencias de escritorio, agentes inteligentes y sistemas de coordinación.

\section{Usabilidad}

La usabilidad de un sistema según Nielsen y Mark es definida como medio para conseguir un objetivo, y que tiene una componente de funcionalidad (utilidad funcional) y otra basada en el modo en que los usuarios pueden usar dicha funcionalidad. La organización ISO nos propone la siguiente definición: La medida en la que un producto se puede usar por determinados usuarios para conseguir objetivos específicos con efectividad, eficiencia y satisfacción en un contexto de uso especificado.

La usabilidad hace referencia a la rapidez y facilidad con que las personas llevan cabo sus tareas propias a través del uso del producto con el que está trabajando, idea que descansa en cuatro puntos:

- Usabilidad significa centrarse en los usuarios.

- Un amplio conocimiento del contexto de uso.

- El producto ha de satisfacer las necesidades del usuario.

- Son los usuarios y no los diseñadores o los desarrolladores, los que determinan cuándo un producto es fácil de usar. 


\section{Métodos para evaluar la usabilidad}

1. Inspección

Los inspectores de la usabilidad pueden ser especialistas en usabilidad, consultores de desarrollo de software con experiencia en guías de estilo de interfaces o usuarios finales que tengan conocimientos de las tareas o del dominio $\mathrm{u}$ otros tipos de profesionales. Se tienen en cuenta las opiniones, juicios, informes de los inspectores sobre elementos específicos de la interfaz como factor fundamental de la evaluación de la usabilidad esto según Nielsen y Mark.

\section{Evaluación heurística}

La evaluación heurística fue desarrollada por Nielsen, 2001 y Molich 1998. La evaluación heurística consiste en analizar la conformidad de la interfaz mediante la inspección de varios evaluadores expertos, se utilizan expertos para validar la interfaz y es posible mejorar perceptiblemente la eficacia del método utilizando a varios evaluadores.

\section{Indagación}

Este tipo de métodos se realiza hablando con los usuarios, observándolos, usando el sistema en trabajo real, u obteniendo respuestas a preguntas por escrito.

\section{Cuestionario}

Tiene la ventaja de llegar a un grupo más numeroso de personas y se puede analizar con más rigor, para esta evaluación se puede utilizar sitios web donde se establece el perfil de usuario (edad, sexo, ocupación, dirección y otros).

\section{Best}

En los métodos de usabilidad por test, usuarios representativos trabajan en tareas utilizando el sistema y los evaluadores utilizan los resultados para ver cómo la interfaz de usuario soporta a los usuarios con sus tareas.

\section{MATERIALES Y MÉTODOS}

\section{Metodología de implementación}

Para apoyar la interacción de grupo se consideró varios aspectos de colaboración. Estos incluyen información compartida, comunicación entre miembros del grupo, y coordinación de las actividades cooperativas.

\section{Desarrollo del sistema}

Se utilizó el ciclo clásico de desarrollo del software, análisis, diseño, implementación y prueba del software.

\section{Software a utilizarse para el desarrollo}

- Macromedia Flash v 7.0: Para realizar las animaciones y herramientas Web.

- Robo demo v 4.0: Para la creación del tutorial de Word.

- Macro media Dreamweare v 6.0, como herramienta de desarrollo del sitio Web.

- PHPv5.1, como herramienta de programación

- Apache v 1.3.37 : Como servidor del sitio de prueba

- MySql v 4: Para la gestión y administración de datos

\section{Curso}

La teleenseñanza se basó en el curso de Microsoft Word el cual se realizó en el periodo de cuatro semanas, en la Universidad Nacional del Callao, en el Centro de Informática de la Facultad de Ingeniería Industrial y de Sistemas.

\section{ELEMENTOS IMPLEMENTADOS PARA EL SISTEMA DE TELEENSEÑANZA}

\section{Plataforma digital}

El sistema de teleenseñanza se implementó con moodle haciendo uso de las tecnologías de programación en PHP, que permite inscripciones de nuevos estudiantes, posibilidad de subir materiales, posibilidad de colocar documentos, posibilidad de acceso a salas de Chat, interactividad (entre docentes estudiantes, entre estudiantes en grupos), acceso a las listas de correos.

\section{Herramienta Web}

Para dar soporte al curso en si, se implementó una herramienta web (visor de animaciones flash) que permite navegar en los módulos del curso.

\section{Materiales y contenidos}

Para esta actividad se diseñaron materiales con soporte de texto y multimedia, utilizándose como herramientas Macromedia Dreamweaver, Macromedia Flash y Robo demo

\section{Modelo de Teleenseñanza}

El proceso de diseño se realizó en dos tramos diferentes y grupos de personas diferentes:

- Un tramo síncrono las actividades fueron en grupos, utilizando la plataforma tecnológica de Groove Networks y el sitio web.

- Un tramo asíncrono, las actividades se realizaron en el sitio web que brinda soporte para diferentes tipos de materiales (texto, multimedia).

\section{Del tramo síncrono}

En esta modalidad se utilizó el software Groove Networks, tuvo una duración de cuatro semanas, se 
realizaron tareas entre profesores y estudiantes, requirió trabajos en el sitio web. Realizándose con la participación de 40 estudiantes de la unidad de Ceups Informática (Ingeniería Industrial, Universidad nacional del Callao) y dos profesores.

El primer encuentro (sitio web) fue entre el profesor (que puede estar en diferente lugar) y los estudiantes (que puede estar en grupos de hasta 10 personas) fue el modelo que se siguió en el tramo síncrono a lo largo del curso. Secuencialmente, el curso en este entorno está constituido por diferentes Momentos:

Momento 1. Esta actividad fue antes del primer encuentro síncrono con Groove Networks y comprendió el proceso de instalación y reconocimiento del entorno, esto se efectuó en el sitio web.

Momento 2. Consistió especialmente en el primer tramo de teleenseñanza con Groove Networks, conviene puntualizar desde un principio que:

- Las clases tuvieron una duración aproximada de 20 minutos.

- Las sesiones tuvieron una duración máxima de 1 hora.

Momento 3. Se utilizó Grove Networks y el apoyo del la herramienta web (visor de animaciones flash) como medio de desarrollo del curso. Este se repitió sucesivamente y que tuvo la característica de mayor proporción de tiempo para intercambios entre los estudiantes y los profesores.

Momento 4, Tramo final. En este momento fue antes de cerrarse la actividad de teleenseñanza en esta modalidad síncrona.

\section{Del tramo asíncrono}

Se realizó el trabajo asíncrono durante cuatro semanas en el sitio web, se emplearon las herramientas web implementados para tal fin, que fueron: área de acceso al curso, área de estudios, área de las evaluaciones, área de grupos, área de materiales.

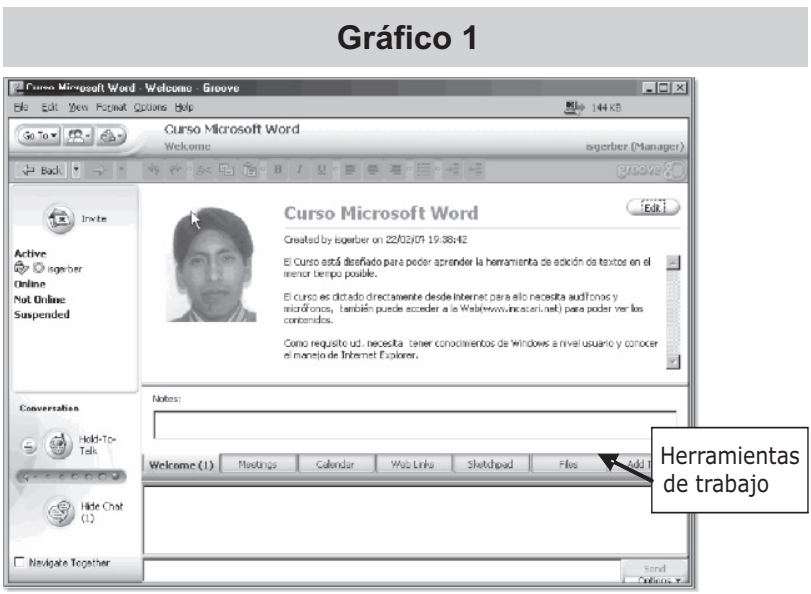

a) Metodología de evaluación del sistema

Se utilizó el test de usabilidad (ver anexo $N^{\circ} 1$ ) tanto para el sistema Groove Networks y el sistema de teleenseñanza en forma independiente y con grupos de personas diferentes en cada caso.

b) Del tratamiento y análisis de datos

Para el tratamiento de la información se tomaron los datos obtenidos en a) y se realizaron el análisis de datos con la estadística descriptiva, tales como cuadros de frecuencias de datos, para ello se utilizaron como herramienta de apoyo el software SPSS versión 14.

\section{EXPOSICIÓNY ANÁLISIS DE RESULTADOS}

\section{Interfaz del espacio de trabajo}

En la Figura 1 se muestra la página de bienvenida al curso de Microsoft Word y en la Figura 2 se muestra el entorno de trabajo con Microsoft Word.

\section{De las invitaciones a la zona de trabajo.}

Una vez que se ha completado el proceso de la reunión para la enseñanza de Microsoft Word, cada uno de los participantes recibirá automáticamente, vía correo electrónico, una invitación. Debido a que Groove Network hace las cosas sin tener en cuenta las preferencias del resto de los miembros, cada usuario obtendrá en dicho mensaje los detalles para poder acceder al curso. El usuario puede llevar a cabo tres acciones: (declinar la invitación o rechazarla; Aceptarla; dejar algún mensaje a los demás invitados, para comunicarle cualquier cambio de planes)

\section{La arquitectura de Groove Networks}

Groove Networks cuenta con la siguiente arquitectura denominada Shared Space Archictecture (ver figura 3). La zona indicada como Transceiver es el enlace con el espacio de trabajo (Shared space) y las cuentas de acceso donde cada uno de ella puede contener un conjunto de herramientas (tools).

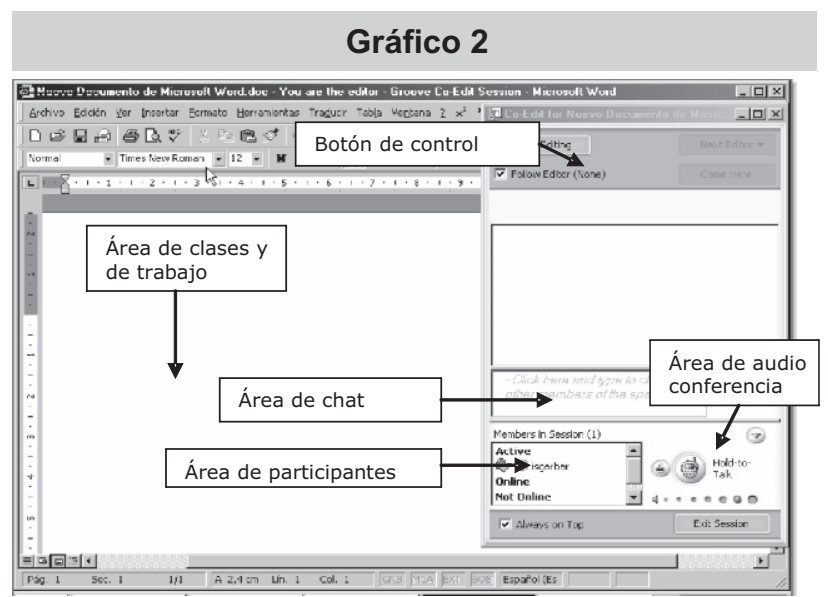




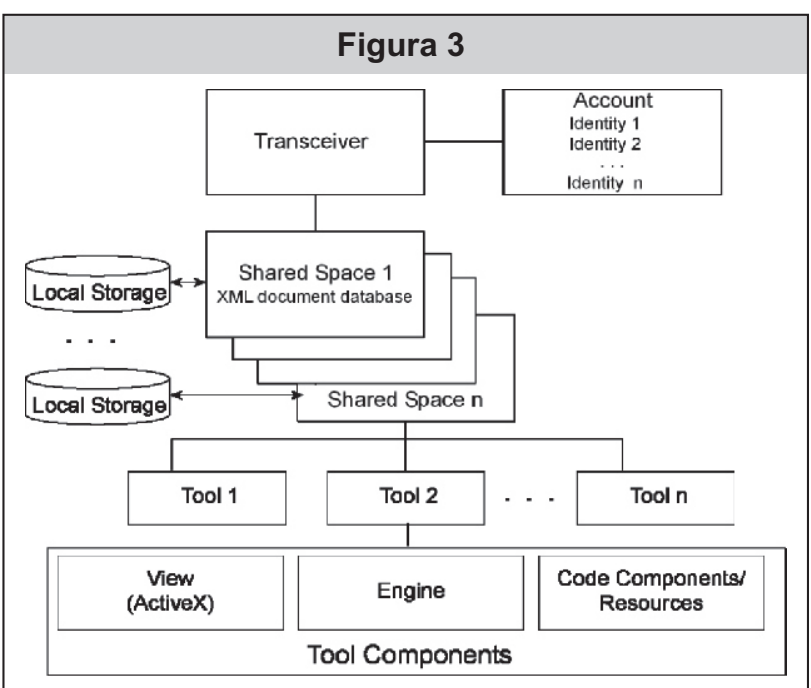

Fuente: Groove Networks 2006

\section{Tipo de conexiones que soporta Groove}

Las conexiones que se puede realizar con Groove pueden ser a través de internet, desde LANs, o desde redes corporativas, tal como se aprecia en la Figura 4.

\section{Problemas identificados utilizando Groove}

- Dificultad para poder acceder al curso

- Dificultad de configuración de micrófonos

- Dificultad para comprender las indicaciones de Groove debido a que utiliza demasiados términos técnicos

Elementos implementados para el sistema de teleenseñanza

\section{- Plataforma digital}

La implementación se realizó con DreamWeaver y utilizando lenguaje PHP como soporte para la gestión de datos.

Una vez en el sistema de enseñanza, se mostrará una pantalla como en la Figura 5.

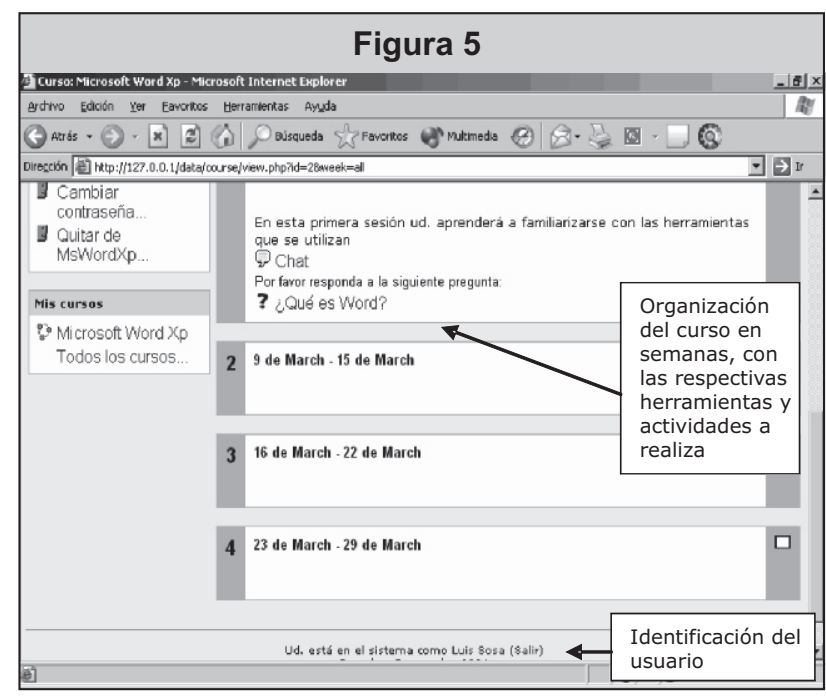

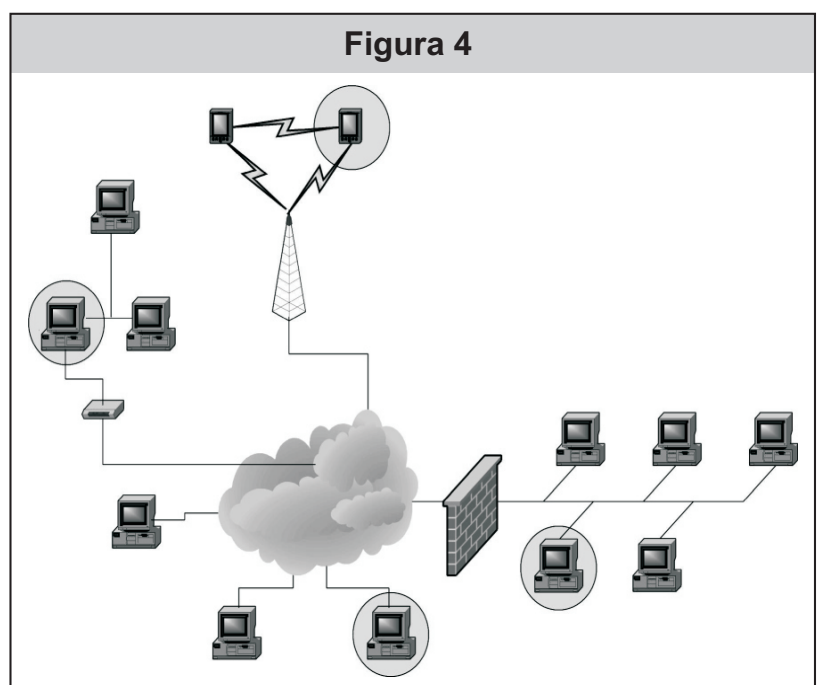

Fuente: Groove Networks 2006

\section{Herramienta Web}

La herramienta web (visor de animaciones flash) fue implementada utilizando Macromedia Flash, que se muestra en la Figura 6.

\section{De la Usabilidad}

Para obtener los cuadros se ha trabajado con una muestra de 40 personas que tienen diferentes niveles de instrucción, usando el test de usabilidad como muestra en las Figuras $7 a$ y $7 \mathrm{~b}$.

Nivel de usabilidad del sistema Groove Networks En el cuadro 2 aprecia que la visibilidad del estado del sistema es bueno en un $35 \%$, seguido de una calificación neutro con el 27,5\%. También se observa que existe una relación regular con respecto del sistema y el mundo real en $40 \%$. Ver cuadro 3 . Con respecto al control del usuario así como la libertad el $50 \%$ realiza una calificación de bueno seguido de una calificación neutro del $37,5 \%$. Ver cuadro 4.

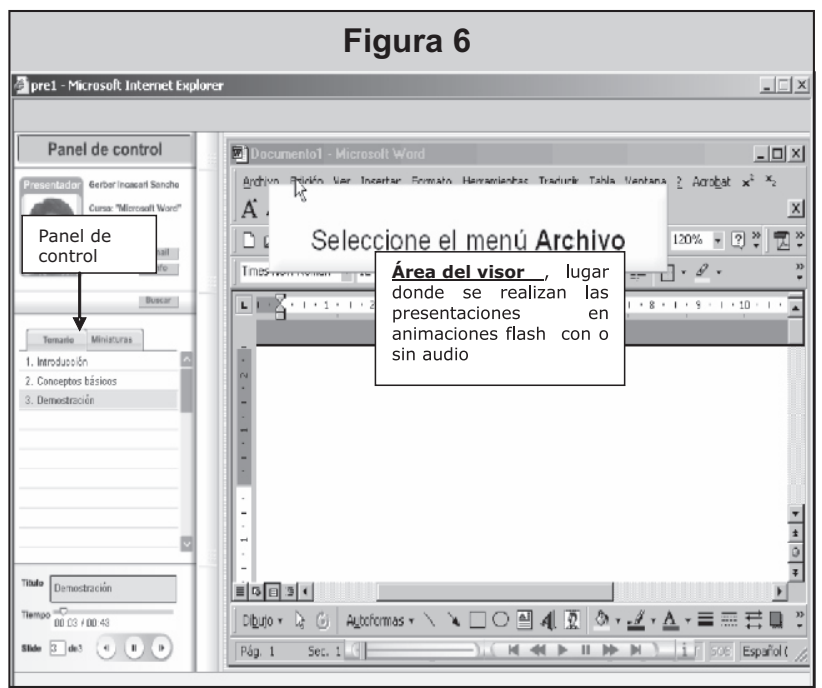


Figura $7 a$

\section{Cuestionario}

\section{Test de usabilidad del sistema}

Edad

Menor a 18

Entre 18 y 25

Entre 25 y 32

Entre 32 y 39

Entre 39 y 46

Mayor de 46

Conocimientos de Internet
Avanzado
Intermedio
Básico

Sexo: Masculino ( )
Estudios

Título Técnico

Título Profesional

Postítulo

Magíster

Doctorado

Lugar e acceso a Internet Navegador que utiliza

Trabajo

Casa

Cabinas

Explore

Netscape

Otros

Conteste UD. las siguientes preguntas

1. ¿̇Ha sido fácil completar la tarea? (Marca la respuesta adecuada)

\begin{tabular}{|c|c|c|c|c|}
\hline Muy fácil & Fácil & Normal & Difícil & Muy difícil \\
\hline
\end{tabular}

2. ¿Compartir información con otros usuarios ha sido?

\begin{tabular}{|c|c|c|c|c|}
\hline Muy fácil & Fácil & Normal & Difícil & Muy difícil \\
\hline
\end{tabular}

3. ¿La información que encontraste UD. en el manual ha sido fácil de utilizar?

\begin{tabular}{|l|l|l|l|c|} 
Muy fácil & Fácil & Normal & Difícil & Muy difícil
\end{tabular}

4. ¿La apariencia del sistema es?

\begin{tabular}{|l|c|c|c|c} 
Excelente & Bueno & Neutro & Regular & Deficiente
\end{tabular}

\section{De la usabilidad}

\begin{tabular}{|l|l|l|l|l|l|}
\hline I. Visibilidad del estado del sistema & $\begin{array}{l}\text { Muy de } \\
\text { acuerdo }\end{array}$ & $\begin{array}{l}\text { De } \\
\text { Acuerdo }\end{array}$ & Neutro & $\begin{array}{l}\text { En } \\
\text { desacuerdo }\end{array}$ & $\begin{array}{l}\text { Muy en } \\
\text { desacuerdo }\end{array}$ \\
\hline $\begin{array}{l}\text { 1.1. El sistema muestra claramente dónde se } \\
\text { encuentra el usuario }\end{array}$ & & & & & \\
\hline 1.2. Las opciones están claramente señalados & & & & & \\
\hline
\end{tabular}

\begin{tabular}{|l|l|l|l|l|l|}
\hline II. Relación entre sistema y mundo real & $\begin{array}{l}\text { Muy de } \\
\text { acuerdo }\end{array}$ & $\begin{array}{l}\text { De } \\
\text { acuerdo }\end{array}$ & Neutro & $\begin{array}{l}\text { En } \\
\text { desacuerdo }\end{array}$ & $\begin{array}{l}\text { Muy en } \\
\text { desacuerdo }\end{array}$ \\
\hline 2.1. El lenguaje es claro & & & & & \\
\hline 2.2. Los conceptos son entendibles & & & & & \\
\hline 2.3. Las palabras son conocidas & & & & & \\
\hline 2.4. Los iconos generan significado & & & & & \\
\hline
\end{tabular}

\begin{tabular}{|l|l|l|l|l|l|}
\hline III. Control del usuario y libertad & $\begin{array}{l}\text { Muy de } \\
\text { acuerdo }\end{array}$ & $\begin{array}{l}\text { De } \\
\text { acuerdo }\end{array}$ & Neutro & $\begin{array}{l}\text { En } \\
\text { desacuerdo }\end{array}$ & $\begin{array}{l}\text { Muy en } \\
\text { desacuerdo }\end{array}$ \\
\hline $\begin{array}{l}\text { 3.1. Es fácil regresar al punto inmediatamente } \\
\text { anterior }\end{array}$ & & & & & \\
\hline $\begin{array}{l}\text { 3.2. Es fácil volver a la pantalla principal desde } \\
\text { cualquier ventana }\end{array}$ & & & & & \\
\hline $\begin{array}{l}\text { 3.3. Provee botones propios para volver o dar } \\
\text { paso a otras pantallas }\end{array}$ & & & & & \\
\hline $\begin{array}{l}\text { 3.4. El sistema soporta varias herramientas de } \\
\text { comunicación }\end{array}$ & & & & & \\
\hline
\end{tabular}

\begin{tabular}{|l|l|l|l|l|l|}
\hline IV. Consiste ncia y estándares & $\begin{array}{l}\text { Muy de } \\
\text { acuerdo }\end{array}$ & $\begin{array}{l}\text { De } \\
\text { acuerdo }\end{array}$ & Neutro & $\begin{array}{l}\text { En } \\
\text { desacuerdo }\end{array}$ & $\begin{array}{l}\text { Muy en } \\
\text { desacuerdo }\end{array}$ \\
\hline $\begin{array}{l}\text { 4.1. Existe coherencia entre el nombre de una } \\
\text { opción y la acción que realiza }\end{array}$ & & & & & \\
\hline 4.2. Todos las opciones tienen contenido & & & & & \\
\hline $\begin{array}{l}\text { 4.3. Existen coherencias entre el título de una } \\
\text { pantalla y su contenido }\end{array}$ & & & & & \\
\hline $\begin{array}{l}\text { 4.4. Sólo existe un botón o enlace que lo lleve } \\
\text { a mismo sitio }\end{array}$ & & & & & \\
\hline
\end{tabular}

\begin{tabular}{|l|l|l|l|l|l|}
\hline V. Prevención de errores & $\begin{array}{l}\text { Muy de } \\
\text { acuerdo }\end{array}$ & $\begin{array}{l}\text { De } \\
\text { acuerdo }\end{array}$ & Neutro & $\begin{array}{l}\text { En } \\
\text { desacuerdo }\end{array}$ & $\begin{array}{l}\text { Muy en } \\
\text { desacuerdo }\end{array}$ \\
\hline $\begin{array}{l}\text { 5.1. Existen mensajes que prevengan posibles } \\
\text { errores }\end{array}$ & & & & & \\
\hline 5.2. Es posible prever posibles errores & & & & & \\
\hline 5.3. La página no induce a cometer errores & & & & & \\
\hline
\end{tabular}


Figura $7 b$

\begin{tabular}{|l|l|l|l|l|l|}
\hline VI. Reconocer en lugar de recordar & $\begin{array}{l}\text { Muy de } \\
\text { acuerdo }\end{array}$ & $\begin{array}{l}\text { De } \\
\text { acuerdo }\end{array}$ & $\begin{array}{l}\text { Neutro } \\
\text { En } \\
\text { Desacuerd } \\
\text { o }\end{array}$ & $\begin{array}{l}\text { Muy en } \\
\text { desacuerd } \\
\text { o }\end{array}$ \\
\hline $\begin{array}{l}\text { 6.1. Los iconos son fácilmente reconocibles } \\
\text { claramente }\end{array}$ & & & & & \\
\hline $\begin{array}{l}\text { 6.3. Es posible reconocer dónde se encuentra el } \\
\text { usuario }\end{array}$ & & & & & \\
\hline
\end{tabular}

\begin{tabular}{|l|l|l|l|l|l|}
\hline VII. Flexibilidad y eficiencia de uso & $\begin{array}{l}\text { Muy de } \\
\text { Acuerdo }\end{array}$ & $\begin{array}{l}\text { De } \\
\text { acuerdo }\end{array}$ & Neutro & $\begin{array}{l}\text { En } \\
\text { desacuerdo }\end{array}$ & $\begin{array}{l}\text { Muy en } \\
\text { desacuerdo }\end{array}$ \\
\hline 7.1. Los opciones son de fácil acceso & & & & & \\
\hline $\begin{array}{l}\text { 7.2. Es fácil agregar herramientas de } \\
\text { comunicación }\end{array}$ & & & & \\
\hline 7.3. Permite el guardado de las acciones & & & & & \\
\hline
\end{tabular}

\begin{tabular}{|l|l|l|l|l|l|}
\hline VIII. Estética y diseño minimalista & $\begin{array}{l}\text { Muy de } \\
\text { acuerdo }\end{array}$ & $\begin{array}{l}\text { De } \\
\text { acuerdo }\end{array}$ & Neutro & $\begin{array}{l}\text { En } \\
\text { desacuerdo }\end{array}$ & $\begin{array}{l}\text { Muy en } \\
\text { desacuerdo }\end{array}$ \\
\hline 8.1. La información es relevante & & & & & \\
\hline 8.2. Contenido bien clasificado & & & & & \\
\hline 8.3. Contenido correctamente organizado & & & & & \\
\hline 8.4. Contenido bien distribuido en el sistema & & & & & \\
\hline
\end{tabular}

IX Reconocimiento, diagnóstico y recuperación de errores

9.1. Es fácil reconocer cuando ocurre un error

9.2. Después que ocurre un error es fácil

volver al sitio de origen

9.3. Cuando ocurre un error existen

mecanismos para solucionarlos

\begin{tabular}{|l|l|l|l|l|l|}
\hline X. Ayuda y documentación & $\begin{array}{l}\text { Muy de } \\
\text { acuerdo }\end{array}$ & $\begin{array}{l}\text { De } \\
\text { acuerdo }\end{array}$ & Neutro & $\begin{array}{l}\text { En } \\
\text { desacuerdo }\end{array}$ & $\begin{array}{l}\text { Muy en } \\
\text { desacuerdo }\end{array}$ \\
\hline 10.1. Existe algún tipo de ayuda & & & & & \\
\hline 10.2. Cuando existe ayuda, ésta es específica & & & & & \\
\hline 10.3. La ayuda está accesible & & & & & \\
\hline
\end{tabular}

Cuestionario de inspección

\begin{tabular}{|l|l|l|l|l|l|}
\hline $\begin{array}{l}\text { ¿Cómo califica UD. globalmente el sistema } \\
\text { analizado? }\end{array}$ & Excelente & Bueno & Neutro & Regular & Deficiente \\
\hline 1. Visibilidad del estado del sistema & & & & & \\
\hline 2. Relación entre sistema y mundo real & & & & & \\
\hline 3. Control del usuario y libertad & & & & & \\
\hline 4. Consistencia y estándares & & & & & \\
\hline 5. Prevención de errores & & & & \\
\hline 6. Reconocer en lugar de recordar & & & & \\
\hline 7. Flexibilidad y eficiencia de uso & & & & \\
\hline 8. Estética y diseño minimalista & & & & \\
\hline 9. Reconocimiento, diagnóstico y recuperación de \\
errores
\end{tabular}

\section{Usabilidad del sitio Web}

Tiene por objetivo evaluar la usabilidad del sitio $W$ eb. Es importante que est e UD. haya explorado y navegado detenidamente por el sitio Web

\begin{tabular}{|l|l|l|l|l|l|}
\hline $\begin{array}{l}\text { ¿Cómo califica globalmente el sitio Web } \\
\text { analizado? }\end{array}$ & $\begin{array}{l}\text { Muy de } \\
\text { acuerdo }\end{array}$ & $\begin{array}{l}\text { De } \\
\text { acuerdo }\end{array}$ & Neutro & $\begin{array}{l}\text { En } \\
\text { desacuerdo }\end{array}$ & $\begin{array}{l}\text { Muy en } \\
\text { desacuerdo }\end{array}$ \\
\hline El sitio Web es fácil de navegar & & & & & \\
\hline Es fácil encontrar la Información deseada & & & & & \\
\hline Los enlaces son claramente identificados & & & & & \\
\hline Los enlaces funcionan correctamente & & & & & \\
\hline $\begin{array}{l}\text { Las páginas se cargan rápidamente (< } \\
\text { segundos) }\end{array}$ & & & & & \\
\hline El uso de las imágenes es aceptable & & & & & \\
\hline El uso del color es aceptable & & & & & \\
\hline El diseño general del sitio es apropiado & & & & & \\
\hline $\begin{array}{l}\text { La organización de la información del sitio es } \\
\text { apropiada }\end{array}$ & & & & & \\
\hline
\end{tabular}


Cuadro 2. Visibilidad del estado del sistema

\begin{tabular}{|c|c|c|c|}
\hline & Frecuencia & Porcentaje \\
\hline & Excelente & 5 & 12,5 \\
\hline & Bueno & 14 & 35,0 \\
\hline & Neutro & 11 & 2,5 \\
\hline n. & Regular & 8 & 20,0 \\
\hline & Deficiente & 2 & 5,0 \\
\hline & Total & 40 & 100,0 \\
\hline
\end{tabular}

Con respecto a la consistencia y estándares se da una calificación de nivel neutro y regular en un $50 \%$ respectivamente (Ver cuadro 5).

El sistema Groove Networks con respecto a la prevención de errores es bueno en $25 \%$, teniendo una calificación de 50\% como neutro (Ver cuadro 6). De la misma manera este sistema permite reconocer en un $42,5 \%$ con un nivel bueno, seguido de un nivel excelente en $37.5 \%$ (Ver cuadro 7).

En el cuadro 8 se puede apreciar que en un $42,5 \%$ tiene una calificación de nivel excelente con respecto a la flexibilidad y eficiencia de uso. Así como el diseño diseño y estética de Groove Networks es bueno en un $67,5 \%$, tal como lo indica el cuadro 9 .

El cuadro 10 muestra que el reconocimiento, diagnóstico y recuperación de errores es bueno en el $50 \%$ de los casos. Con respecto a la ayuda y documentación se observa que el mayor porcentaje obtenido es en el nivel regular en un $50 \%$ seguido del nivel neutro en $30 \%$ (Ver cuadro 11).

\section{Nivel de usabilidad del sistema teleenseñanza}

En el cuadro 12 se aprecia que el sistema de teleenseñanza es un sitio web fácil de navegar en $47.5 \%$, mientras el cuadro 13 indica que en un nivel neutro de $52,2 \%$ con respecto a la facilidad de encontrar la información deseada.

En el cuadro 14 se puede apreciar que el sitio web tiene un nivel muy de acuerdo en $47,5 \%$ en lo

Cuadro 4. Control del usuario y libertad

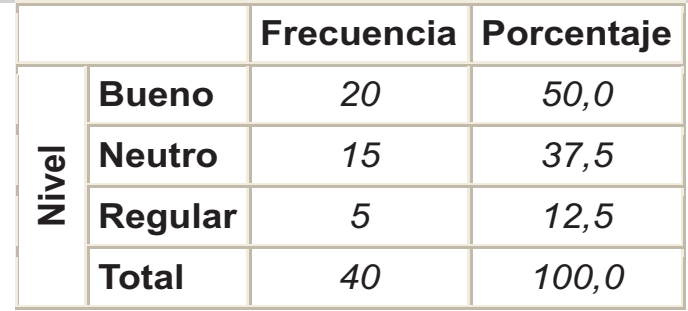

Cuadro 3. Relación entre el sistema y el mundo real

\begin{tabular}{|c|c|c|c|}
\hline & Frecuencia & Porcentaje \\
\hline \multirow{6}{*}{$\sum_{\bar{z}}^{\bar{\Phi}}$} & Excelente & 1 & 2,5 \\
\hline & Bueno & 10 & 25,0 \\
\hline & Neutro & 11 & 27,5 \\
\hline & Regular & 16 & 40,0 \\
\hline & Deficiente & 2 & 5,0 \\
\hline & Total & 40 & 100,0 \\
\hline
\end{tabular}

referente a la claridad de los enlaces. Asimismo el $62,5 \%$ considera estar de acuerdo con el funcionamiento de los enlaces son correctos (Ver cuadro 15).

El $45 \%$ considera neutro con respecto a la velocidad de carga de páginas y el $35 \%$ esta en desacuerdo, es decir la carga de páginas supera los 30 segundos (Ver cuadro 16).

El cuadro 17 indica que el $27,5 \%$ mencionan estar de muy de acuerdo con el uso de las imágenes. Asimismo se observa que el $45 \%$ esta de acuerdo con el diseño general, encontrándose así mismo muy en desacuerdo el $5 \%$ (Ver cuadro 18).

El cuadro 19 muestra que la organización de la información del sitio se considera neutro en un $42.5 \%$, el $30 \%$ menciona estar de acuerdo y el cuadro 20 indica con respecto a la relevancia del sitio web es considerado dentro del nivel de acuerdo y muy de acuerdo en $40 \%$ respectivamente.

El cuadro 21 indica que el 55\% considera que la interfaz del sitio es placentera en un nivel de acuerdo. mientras que en el cuadro 22 se muestra que el $70 \%$ considera estar muy de acuerdo con las funcionalidades implementadas.

El cuadro 23 indica que el $42,5 \%$ consideran en un nivel neutro las capacidades esperadas, el $30 \%$ considera estar de acuerdo. Finalmente El cuadro 24 indica que el $70 \%$ considera estar de acuerdo con el uso del color del sitio web.

Cuadro 5.: Consistencia y estándares

\begin{tabular}{|c|l|c|c|}
\hline \multicolumn{2}{|c|}{} & Frecuencia & Porcentaje \\
\hline \multirow{2}{*}{$\sum_{\overline{\mathbf{Z}}}^{\mathbf{\Phi}}$} & Neutro & 20 & 50,0 \\
\cline { 2 - 4 } & Regular & 20 & 50,0 \\
\cline { 2 - 4 } & Total & 40 & 100,0 \\
\hline
\end{tabular}


Cuadro 6: Prevención de errores

\begin{tabular}{|c|c|c|c|}
\hline & Frecuencia & Porcentaje \\
\hline \multirow{4}{*}{$\sum_{\bar{z}}^{\bar{\Phi}}$} & Bueno & 10 & 25,0 \\
\hline & Neutro & 20 & 50,0 \\
\hline & Regular & 10 & 25,0 \\
\hline & Total & 40 & 100,0 \\
\hline
\end{tabular}

Cuadro 8. Flexibilidad y eficiencia de uso

\begin{tabular}{|c|c|c|c|}
\hline & Frecuencia & Porcentaje \\
\hline \multirow{6}{*}{$\sum_{\bar{z}}^{\bar{\Phi}}$} & Excelente & 17 & 42,5 \\
\hline & Bueno & 8 & 20,0 \\
\hline & Neutro & 4 & 10,0 \\
\hline & Regular & 7 & 17,5 \\
\hline & Deficiente & 4 & 10,0 \\
\hline & Total & 40 & 100,0 \\
\hline
\end{tabular}

Cuadro 10. Reconocimiento, diagnóstico y recuperación de errores

\begin{tabular}{|c|l|c|c|}
\hline \multicolumn{2}{|c|}{} & Frecuencia & Porcentaje \\
\hline \multirow{2}{*}{ Nivel } & Bueno & 20 & 50,0 \\
\cline { 2 - 4 } & Neutro & 20 & 50,0 \\
\cline { 2 - 4 } & Total & 40 & 100,0 \\
\hline
\end{tabular}

Cuadro 12. El sitio web es fácil de navegar

\begin{tabular}{|c|c|c|c|}
\hline & Frecuencia & Porcentaje \\
\hline \multirow{5}{*}{$\sum_{\bar{z}}^{\bar{\Phi}}$} & Muy de acuerdo & 2 & 5,0 \\
\hline & De acuerdo & 19 & 47,5 \\
\hline & Neutro & 9 & 22,5 \\
\hline & En desacuerdo & 10 & 25,0 \\
\hline & Total & 40 & 100,0 \\
\hline
\end{tabular}

Cuadro 14. Los enlaces son claramente identificados

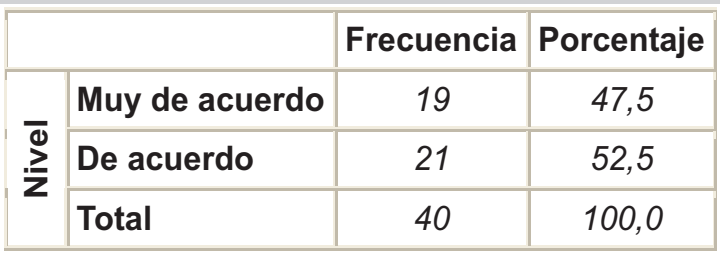

Cuadro 7: Reconocer en lugar de recordar

\begin{tabular}{|c|c|c|c|}
\hline & Frecuencia & Porcentaje \\
\hline \multirow{5}{*}{$\frac{\bar{\Phi}}{\bar{z}}$} & Excelente & 15 & 37,5 \\
\hline & Bueno & 17 & 42,5 \\
\hline & Neutro & 2 & 5,0 \\
\hline & Regular & 6 & 15,0 \\
\hline & Total & 40 & 100,0 \\
\hline
\end{tabular}

Cuadro 9. Estética y diseño minimalista

\begin{tabular}{|c|c|c|c|}
\hline & Frecuencia & Porcentaje \\
\hline \multirow{5}{*}{$\sum_{\frac{\Phi}{\mathbf{Q}}}^{\bar{z}}$} & Bueno & 27 & 67.5 \\
\hline & Neutro & 8 & 20.0 \\
\hline & Regular & 4 & 10.0 \\
\hline & Deficiente & 1 & 2.5 \\
\hline & Total & 40 & 100.0 \\
\hline
\end{tabular}

Cuadro 11. Ayuda y documentación

\begin{tabular}{|c|l|c|c|}
\hline \multicolumn{2}{|l|}{} & Frecuencia & Porcentaje \\
\hline \multirow{2}{*}{$\bar{\Phi}$} & Neutro & 12 & 30,0 \\
\cline { 2 - 4 } & Regular & 20 & 50,0 \\
\cline { 2 - 4 } $\mathbf{Z}$ & Deficiente & 8 & 20,0 \\
\cline { 2 - 4 } & Total & 40 & 100,0 \\
\hline
\end{tabular}

Cuadro 13. Es fácil de encontrar la información deseada

\begin{tabular}{|c|c|c|c|}
\hline & & Frecuencia & Porcentaje \\
\hline \multirow{5}{*}{$\sum_{z}^{\bar{\phi}}$} & De acuerdo & 8 & 20,0 \\
\hline & Neutro & 21 & 52,5 \\
\hline & En desacuerdo & 9 & 22,5 \\
\hline & Muy en desacuerdo & 2 & 5,0 \\
\hline & Total & 40 & 100,0 \\
\hline
\end{tabular}

Cuadro 15. Los enlaces funcionan correctamente

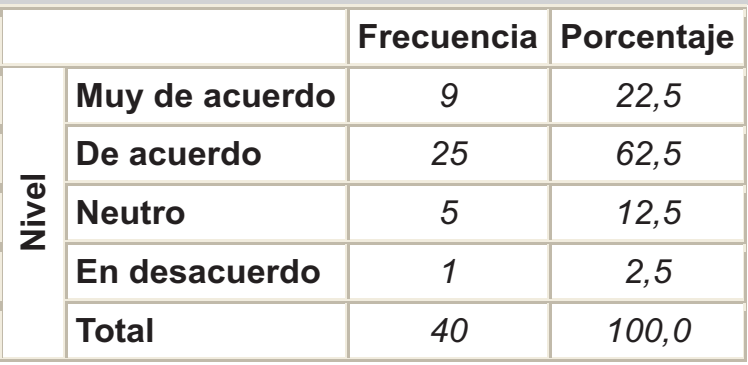


Cuadro 16. Las páginas se cargan rápidamente

\begin{tabular}{|l|l|c|c|}
\multicolumn{2}{|l|}{} & Frecuencia & Porcentaje \\
\hline $\bar{\Xi}$ & De acuerdo & 8 & 20,0 \\
\hline \multirow{2}{\mathbf{Z}}{} & Neutro & 18 & 45,0 \\
\hline En desacuerdo & 14 & 35,0 \\
\hline & Total & 40 & 100,0 \\
\hline
\end{tabular}

Cuadro 18. El diseño general del sitio es apropiado

\begin{tabular}{|l|l|c|c|}
\hline \multicolumn{2}{|l|}{} & Frecuencia & Porcentaje \\
\hline \multirow{2}{*}{$\bar{D}$ De acuerdo } & 18 & 45,0 \\
\hline \multirow{2}{*}{$\overline{\mathbf{J}}$ Neutro } & 14 & 35,0 \\
\cline { 2 - 4 } & En desacuerdo & 6 & 15,0 \\
\cline { 2 - 4 } & Muy en desacuerdo & 2 & 5,0 \\
\hline \multirow{2}{*}{ Total } & 40 & 100,0 \\
\hline
\end{tabular}

Cuadro 20. El contenido del sitio es relevante

\begin{tabular}{|l|l|c|c|}
\hline \multicolumn{2}{|l|}{} & Frecuencia & Porcentaje \\
\hline & Muy de acuerdo & 16 & 40,0 \\
\hline \multirow{2}{*}{$\bar{D}$ De acuerdo } & 16 & 40,0 \\
\hline \multirow{2}{\mathbf{Z}}{ Neutro } & 6 & 15,0 \\
\hline En desacuerdo & 2 & 5,0 \\
\hline & Total & 40 & 100,0 \\
\hline
\end{tabular}

Cuadro 22. El sitio tiene todas las funcionalidades esperadas

\begin{tabular}{|c|l|c|c|}
\hline \multicolumn{2}{|l|}{} & Frecuencia & Porcentaje \\
\hline \multirow{2}{*}{$\bar{\Phi}$} & Muy de acuerdo & 28 & 70,0 \\
\cline { 2 - 4 } $\mathbf{z}$ & De acuerdo & 12 & 30,0 \\
\cline { 2 - 4 } & Total & 40 & 100,0 \\
\hline
\end{tabular}

Cuadro 17. El uso de imágenes es aceptable

\begin{tabular}{|l|l|c|c|}
\hline \multicolumn{2}{|l|}{} & Frecuencia & Porcentaje \\
\hline & Muy de acuerdo & 11 & 27,5 \\
\hline De acuerdo & 8 & 20,0 \\
\hline \multirow{\Xi}{\mathbf{z}}{} & Neutro & 10 & 25,0 \\
\hline En desacuerdo & 5 & 12,5 \\
\hline Muy en desacuerdo & 6 & 15,0 \\
\hline Total & 40 & 100,0 \\
\hline
\end{tabular}

Cuadro 19. La organización de la información del sitio web es apropiada

\begin{tabular}{|l|l|c|c|}
\hline \multicolumn{2}{|l|}{} & Frecuencia & Porcentaje \\
\hline & Muy de acuerdo & 1 & 2,5 \\
\hline De acuerdo & 12 & 30,0 \\
\hline \multirow{\Xi}{\mathbf{\Xi}}{} & Neutro & 17 & 42,5 \\
\hline En desacuerdo & 10 & 25,0 \\
\hline Total & 40 & 100,0 \\
\hline
\end{tabular}

Cuadro 21. La interfaz del sitio es placentera

\begin{tabular}{|c|c|c|c|}
\hline & & Frecuencia & Porcentaje \\
\hline \multirow{5}{*}{$\stackrel{\bar{\Xi}}{\frac{\Phi}{z}}$} & Muy de acuerdo & 4 & 10,0 \\
\hline & De acuerdo & 22 & 55,0 \\
\hline & Neutro & 11 & 27,5 \\
\hline & En desacuerdo & 3 & 7,5 \\
\hline & Total & 40 & 100,0 \\
\hline
\end{tabular}

Cuadro 23. El sitio tiene todas las capacidades esperadas

\begin{tabular}{|c|l|c|c|}
\hline \multicolumn{2}{|c|}{} & Frecuencia & Porcentaje \\
\hline \multirow{2}{*}{$\bar{\Phi}$} & De acuerdo & 12 & 30,0 \\
\cline { 2 - 4 } & Neutro & 17 & 42,5 \\
\cline { 2 - 4 } $\mathbf{z}$ & En desacuerdo & 11 & 27,5 \\
\cline { 2 - 4 } & Total & 40 & 100,0 \\
\hline
\end{tabular}

\begin{tabular}{|c|c|c|c|}
\hline \multicolumn{4}{|c|}{ Cuadro 24. El uso del color es aceptable } \\
\hline & & Frecuencia & Porcentaje \\
\hline \multirow{5}{*}{$\sum_{\frac{1}{z}}^{\bar{\Phi}}$} & Muy de acuerdo & 1 & 2,5 \\
\hline & De acuerdo & 28 & 70,0 \\
\hline & Neutro & 8 & 20,0 \\
\hline & En desacuerdo & 3 & 7,5 \\
\hline & Total & 40 & 100,0 \\
\hline
\end{tabular}




\section{CONCLUSIONES}

El sistema colaborativo Groove Networks presenta niveles de usabilidad de "bueno" en cinco de los casos con porcentajes de van desde $50 \%$ a $67 \%$, seguidos de nivel "regular" con porcentajes que van desde $40 \%$ a $50 \%$.

El sistema de teleenseñanza presenta los siguiente niveles de usabilidad "de acuerdo" en seis de los casos con porcentajes que van desde $42.5 \%$ hasta $70 \%$, seguidos por el nivel "neutro" en cuatro de los casos que tiene un rango en porcentajes de van desde $41.5 \%$ hasta $52.5 \%$.

De los resultados se determina, que el sistema colaborativo Grove Networks tiene mayor porcentaje de un nivel "bueno" que es un segundo nivel dentro de la categoría analizada(cinco niveles), frente a un nivel "de acuerdo" en el sistema de teleenseñanza que ocupa el segundo nivel de cinco niveles de usabilidad, por otra parte de los resultados Groove Netoworks al ser una herramienta de espacio compartido tiene el $42.5 \%$ en nivel de "excelente" esto se explica debido a la flexibilidad y eficiencia de uso.

\section{REFERENCIAS BIBLIOGRAFICAS}

1. RODRIGUEZ ROSELLÓ, L. 1999. Investigación y desarrollo en la Comunidad Europea sobre tecnologías para el aprendizaje: Status y perspectivas. Infodidac, pp. 98.

2. JAY, A., 1993. "How to Run a Meeting", Readings in Groupware and Computer-Support Cooperative Work, San Mateo, CA, Morgan Kaufmann Publishers, pp.130-145.

3. ELLIS, C.A., Gibbs, S J., Rein, G.L.: 2001. "Groupware some issues y experiences", Comm. of the ACM, Vol. 34 No. 1, pp. 38-58.

4. NIELSEN J. y MACK R. L. 2001.eds. Usability Inspection Methods. John Wiley and Sons, New York, NY.

5. MOLICH R. y NIELSEN J. 1998. Heuristic evaluation of user interfaces en Proceedings of ACM CHI 1990. Seattle, pp. 249-256.

6. ALCALDE, E. y GARCÍA, J. 1993. Introducción a la teleinformática. McGraw-Hill. Madrid.

7. CARPIO I., J. La Red Educativa de Videoconferencia de la UNED. España. 2000.

8. GRUDIN, J. 1994.Computer Supported Cooperative Work: History and Focus, Computer, Vol. 27, No. 5, pp. 19-26.

9. SEVILLANO G., M.L. 2002. La Producción de Medios Audiovisuales en la UNED, A Distancia, $\mathrm{N}^{\circ}$ de Primavera. pp. 95, España Madrid. 